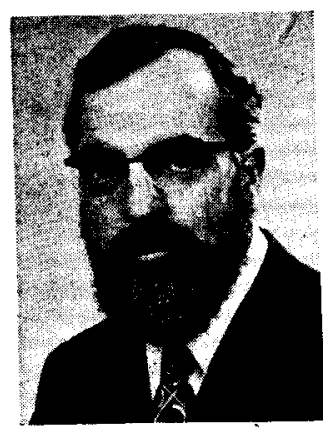

\title{
Lasers and Optical Electronics
}

\author{
Professor W. A. GAMBLING, D.Sc., Ph.D., C.Eng., F.I.E.E., F.I.E.R.E. \\ Quantum electronics led to the development of stimulated emission devices, first \\ the maser and then the laser. The latter has made possible many applications in \\ optical electronics, the most important of which are in communication using optical \\ fibres.
}

\section{Introduction}

The trend in electronics has always been to shorter time scales and higher frequencies, for the simple reason that only by such means can the rate of handling and transmission of information be increased. Lurking behind this argument, of course, is the none-too-subtle and dominating influence of economics, because by-andlarge it is usually cheaper to produce a new system of higher capacity than to duplicate existing ones. If this were not so then there would be no point in employing frequencies higher than, say, $1 \mathrm{MHz}$ since one might make a $100 \mathrm{MHz}$ link by using a hundred $1 \mathrm{MHz}$ systems in parallel. This is obviously not an economically practicable solution!

In the early days of electronics, operating frequencies of triodes and pentodes were gradually pushed up into the megahertz region until the effects of electron transit time and stray capacitance became dominant. Then came a period of technological trickery and ingenuity as devices were pushed to their limits, culminating in the development of coaxial triodes with grid/cathode spacings of 10 to $20 \mu \mathrm{m}$ but it was obvious that a radically new technique was required before any further large advance could be achieved. This came about, as it were, by the acceptance of the adage 'If you can't beat

Professor Alec Gambling (Fellow 1964, Member 1958) has held the Chair in Electronics at the University of Southampton since 1964 and has been Head of the Department since 1974 $\mathrm{He}$ is at present also Dean of the Faculty of Engineering and Applied Science. Professor Gambling graduated in Electrical Engineering from the University of Bristol; his Ph.D. was awarded by the University of Liverpool and his D.Sc. by the University of Bristol. Before going to Southampton, Professor Gambling was a lecturer at the University of Liverpool and he also spent two years as a postdoctoral research fellow at the University of British Columbia. He has held appointments as visiting professor at the Bhabha Atomic Research Centre, Bombay, and at the University of Colorado.

$\mathrm{He}$ is a member of the Optics and Infra-Red Committee of the Electronics Research Council and of the Technology Sub-Committee of the UGC.

Actively involved in Institution affairs for many years, Professor Gambling has served as Chairman of the Southern Section Committee, on the Education Committee and as an Ordinary Member of the Council; he was a Vice-President from $1970-1973$ and from 1974.

Professor Gambling has contributed numerous original papers and reviews to the technical literature on a wide range of subjects, his main research interests being in microwaves, lasers, and more recently optical-fibre communications. 'em, join 'em', and instead of allowing the finite transit time to remain a limitation it was put to use, firstly in the klystron, in order to produce bunching and thus amplification. Klystrons are nonetheless limited by the transit time through the resonator gap and thus the true distributed interaction device, the travelling-wave tube (and the associated backward-wave oscillator), were later invented and are still widely used in microwave and satellite communications. However, even these are limited, by the difficulty in fabricating the associated slow-wave structure, to frequencies in the region of $10 \mathrm{GHz}$ although some superb precision engineering produced the carcinotron operating at nearly $1000 \mathrm{GHz}$.

Developments in solid-state devices followed a similar path with the limitations of transit time and capacitance in transistors eventually being circumvented in transferred-electron devices such as Gunn diodes and the like, which in turn are limited by fabrication difficulties.

\section{Quantum Electronics}

Another 'breakthrough' was therefore required and came about via quantum electronic devices, firstly the maser $\dagger$ and then the laser. Since they do not depend on an associated structure (other than the mirrors) lasers are true distributed-interaction devices. Again their development followed a classic pattern in that the basic theory was enunciated by Einstein ${ }^{\mathbf{1}}$ in 1917, then largely ignored, and it was not until 1954 that the first successful device was operated. Interestingly enough this time delay is even longer than that between the prediction of electromagnetic waves by Maxwell in 1864 and their first demonstration by Hertz 23 years later.

The history of quantum electronics throws a very interesting light (if the pun may be excused) on the interplay between electronics and physics and on how pure and applied science are inextricably intertwined so that they complement and depend one upon the other. During the 1939-45 war scientists from many different disciplines were recruited to help in the development of radar and, as a result, became expert in electronic and microwave techniques. After the war they applied their new-found expertise to their own fields, notably in

+ The device is so named from its description $M$ icrowave Amplification by the Stimulated Emission of Radiation (not, as was sometimes unkindly claimed at the time, Means of $A$ cquiring Support for Expensive Research). In laser the initial letter stands for Light. 
microwave spectroscopy, giving rise to such distinguished research schools as that at Oxford. This powerful and novel method of investigation led to an explosion of knowledge relating to atomic and molecular structure so that technology's debt to science was amply repaid. The story does not end there as an extension of microwave spectroscopy produced the maser which engineers developed into a new and better frequency standard and into the most sensitive amplifier ever made, leading in turn to the first satellite communication system. Maser amplifiers also increased the sensitivity of radio telescopes and led to new fundamental knowledge about the stars. A similar interplay between technology and science has occurred with the laser.

\section{Stimulated Emission}

As mentioned above, the basic theory of stimulated emission, on which laser action depends, was first enunciated by Einstein by some brilliant deductive reasoning, and is disarmingly simple. Many types of atom and molecule can exchange energy with an electromagnetic field and since atoms can have only certain discrete values of internal energy, and the electromagnetic field is quantized, the frequency $f$ which the radiation must have in order that interaction occurs is related to the change in energy $\Delta E$ in a very simple way, namely

where $h$ is Planck's constant.

$$
\Delta E=h f
$$

There are three types of interaction process and the simplest of these to understand is absorption. If radiation of frequency $f_{12}$ passes through an assembly of atoms having an energy $E_{1}$ and capable of absorbing energy so that their internal energy rises to $E_{2}$, where

$$
E_{2}-E_{1}=h f_{12}
$$

then the number of photons absorbed per second is simply proportional to the density of the radiation $u$ (i.e. to the number of photons) and to the number $N_{1}$ of atoms present in energy state $E_{1}$, thus

$$
\frac{\mathrm{d} N_{1}}{\mathrm{~d} t}=-B_{12} u N_{1}
$$

where $B_{12}$ is a constant.

A second process is that of spontaneous emission which, like radioactive decay, is a random process. In this case if the number of atoms present having an energy $E_{2}$ is $N_{2}$ then photons of energy $h f_{12}$ are emitted even in the absence of an external source of radiation and the rate of emission is proportional to $N_{2}$ so that

$$
\frac{\mathrm{d} N_{1}}{\mathrm{~d} t}=A N_{2}
$$

These two processes are well known and are easily understood but Einstein's contribution was to show, from thermodynamic considerations, that there is a third process involving an inverse mechanism to absorption of radiation. Thus atoms of energy $E_{2}$ can be stimulated to emit photons by other photons of energy $h f_{12}$ and the emission rate, as in absorption, is proportional to the number of stimulating photons (i.e. radiation density $u$ ) and to the number $N_{2}$ of atoms capable of emitting, whence

$$
\frac{\mathrm{d} N_{1}}{\mathrm{~d} t}=B_{21} u N_{2}
$$

Hence in an assembly where all three processes occur and containing $N_{1}$ atoms having energy $E_{1}$ and $N_{2}$ with energy $E_{2}$, then the net emission rate of photons is given by

$$
\frac{\mathrm{d} N_{1}}{\mathrm{~d} t}=B u\left(N_{2}-N_{1}\right)+A N_{2}
$$

since it turns out that $B_{12}=B_{21}$.

The first term in (5) represents amplification, if $N_{2}>N_{1}$, because the stimulated emission is coherent, and in phase, with the stimulating emission. On the other hand, the spontaneous emission term represents noise due to the random nature of the process.

In the blinding light of hindsight it seems incredible that there was such a long time-lapse between the development of Einstein's theory and its practical demonstration. Indeed some electrical discharges in gases can produce stimulated emission so easily that it would not be surprising for it to have been observed accidentally very much earlier. One wonders whether the effect manifested itself in an experiment only to be dismissed as an anomalous result. This brings to mind the (apocryphal?) story concerning the discovery of $\mathrm{X}$-rays by Roentgen who observed that photographic plates stored near a high-voltage cathode-ray machine were blackened and his curiosity was aroused. It is also said that at about the same time a technician who informed the Professor of Experimental Philosophy at the University of Oxford that photographic plates stored near his cathode-ray machine were blackened, was told to store them somewhere else. A sensible reply perhaps but not an inquisitive one.

The great difficulty in achieving amplifying conditions is, of course, that of achieving at least a quasi-stable condition in any system with $N_{2}>N_{1}$ because, in thermal equilibrium, $N_{1}$ always exceeds $N_{2}$ as required by the Boltzmann relation:

$$
\frac{N_{2}}{N_{1}}=\exp \left(-\frac{h f_{12}}{k T}\right)
$$

The first clear recognition of the possibility of amplification of electromagnetic radiation by stimulated emission seems to have been by a Russian, Fabrikant, ${ }^{2}$ who filed a patent in 1951 (although it was not published until 1959) and who had discussed various aspects in his thesis of 1940. However, his attempts to produce optical amplification in caesium were unsuccessful.

The first statement in the open literature about amplification was by $\mathrm{Weber}^{3}$ in 1953 , followed by the detailed proposals of Basov and Prokhorov ${ }^{4}$ for a beam-type maser in 1954. However, the real excitement was caused by the short article of Gordon, Zeiger and Townes, ${ }^{5}$ in the same year, announcing the operation of the first maser using ammonia. Townes had conceived the required experimental arrangement three years earlier, based on his experience in microwave spectroscopy. In the years immediately following many other techniques were studied, but the only one to give any 

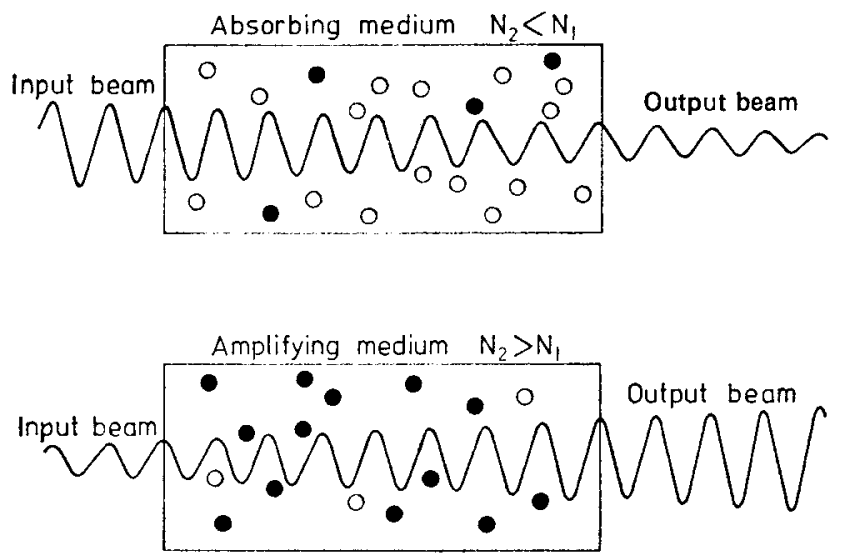

Fig. 1. Interaction of radiation with an atomic system. In the upper diagram energy is absorbed from the input beam because of the preponderance of atoms in the lower energy state (O). The lower diagram indicates the situation where there are more atoms capable of stimulated emission (O) than there are of absorbing radiation with the result that the input beam is amplified.

degree of practical success was the three-level maser of Bloembergen ${ }^{6}$ which resulted in the ruby maser amplifier. The ammonia maser, now almost forgotten, proved to be a microwave oscillator $(23.9 \mathrm{GHz})$ of very high stability, although the hydrogen maser is now preferred. It has only once been used as an amplifier, by Wilmshurst and Gambling, ${ }^{7}$ because of its very narrow bandwidth $(\sim 300 \mathrm{~Hz})$ and low saturation power $\left(10^{-12} \mathrm{~W}\right)$.

\section{The Laser}

Because of the great interest aroused by masers it was not until 1957 that further serious attention was given to the idea of producing an optical version of the maser.

In their classic article of 1958 on the principles of laser action Shawlow and Townes ${ }^{8}$ suggested potassium vapour as a possible medium and much effort was devoted to it but with no success. The reasons for this failure were rather puzzling, especially as other workers later found caesium vapour to behave as predicted. Another medium under consideration was ruby $\left(\mathrm{Cr}^{3+}\right.$ in $\mathrm{Al}_{2} \mathrm{O}_{3}$ ) although an internal report at Bell Telephone Laboratories concluded that the existing material was much too poor to give any hope of success and the experts of the time expected that the first laser would be based on a gas. Great was the surprise and general jubilation therefore when Maiman, who had persevered with ruby, achieved laser action in 1960 . Maiman's own jubilation was short-lived as the manuscript which he prepared announcing his remarkable result was rejected by Physical Review Letters and an historic scoop of scientific journalism was achieved by the journals Nature and British Communications and Electronics which carried the first announcement ${ }^{9}$ in the established scientific literature.

Some months later the helium/neon laser was successfully operated and there followed over the next few years a tremendous explosion of publications on laser transitions in hundreds of different materials and on the properties of laser devices. The author well remembers the 3rd International Quantum Electronics Conference in Paris where, for a week, there were parallel sessions each day lasting from 0900 hours to nearly 1900 , with more than 200 presentations and 1,100 participants. The pace of development was so rapid that two consecutive papers on gas lasers from the same organization were presented with the respective groups of authors not knowing the results of the others until they were read out. Those were heady days and one wonders whether we shall ever again see so much fundamental research done on such a fruitful topic.

Many thousands of papers and lasing transitions have appeared since 1960 and in addition to gas and solidstate lasers we have liquid, dye and semiconductor devices operating at fixed wavelengths from the submillimetre to the ultraviolet regions of the spectrum. The number of wavelengths at which laser operation has been achieved runs into many thousands, but only a relatively small number of lasers are manufactured on any scale. Applications are, to some extent, limited by the comparatively few wavelengths easily available, and the thought of having to develop a tailor-made device is a large deterrent so that the appearance of a number of tunable laser sources is of considerable significance.

\section{Properties of Laser Radiation}

The laser is essentially an electronic oscillator of extremely high frequency. Structurally it differs from most lower-frequency oscillators in its highly-multimode resonator, so that although it is possible to obtain, relatively easily, a Gaussian beam it is normally composed of a number of frequency components. For most applications this is no problem and if necessary a single axial mode can be selected by means of a resonant reflector.

The difference between laser, or coherent, radiation and incoherent light such as that emitted from ordinary light sources is similar to the difference between a sinusoidal signal and electrical noise. Electronic techniques would be in a very primitive state if the only sources available were noise generators and yet this was the case in optical electronics until the laser appeared.

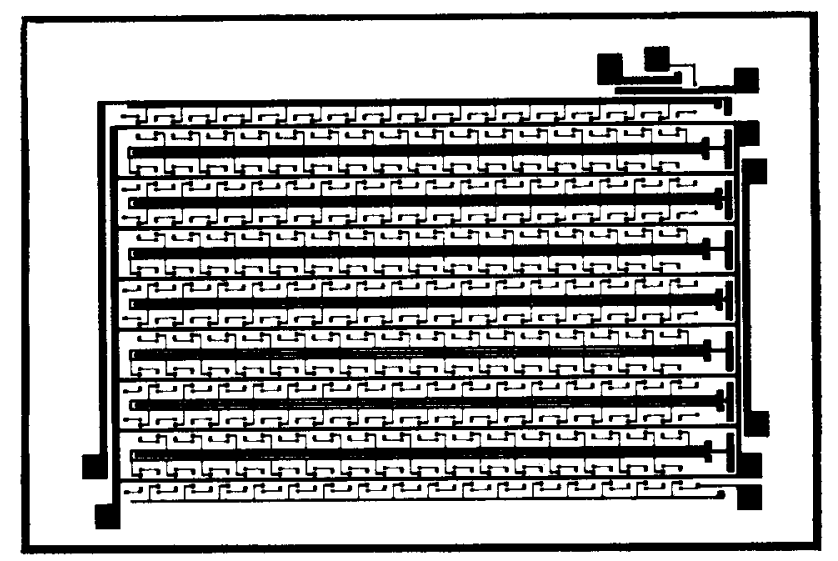

Fig. 2. Microelectronic circuit mask for a molybdenum-gate auto-registered m.o.s. shift register produced by laser machining. (By courtesy of Professor K. G. Nichols, Department of Electronics, University of Southampton.) 
Before anything like the same development can take place at optical frequencies, as we take for granted in conventional electronics, we must learn to use the new devices and to produce the required optical components and systems. Significant progress is now being made in this direction.

The properties of laser light which make it potentially so useful are simple and obvious. For example, it can be collimated to a degree limited only by diffraction, whereby the half-angle spread $\theta$ of a beam produced by an aperture $D$ is related to the wavelength $\lambda$ by $\theta \simeq \lambda / D$. Thus a helium/neon laser giving a beam of diameter $2 \mathrm{~mm}$, for which $\theta \sim 0.3 \mathrm{mrad}$, forms a cheap and simple light pencil which has found a score of uses in many diverse fields. A list of laser applications would be quite out of place here but one can perhaps mention a few. Simple lasers are now standard equipment on tunnel-boring machines which can be programmed to keep themselves aligned on the beam thus obviating the tedious and time-consuming surveying techniques. A more homely application is reflected in a ten-milliondollar order recently received by the Spectra Physics Corporation in the USA for laser scanning equipment to be used at supermarket cash desks. The checker simply moves the items, which are suitably marked, across the beams in a simple, fast, natural motion. The scanning equipment then records the price, operates the till, alters the store inventory and provides an itemized tape for the customer.

In addition to collimation a coherent beam can be focused by a lens of focal length $f$ to a spot of diameter $d \sim f \lambda / D$. Thus if $f \simeq D$ then $d \simeq \lambda$ and power den- sities can be achieved which are capable of melting and vaporizing any known material. Obviously in most cases mechanical drilling is still the simplest and cheapest method but with refractory, brittle, poisonous or highpurity materials, or where precision and minimum effect on surrounding areas are important, laser machining comes into its own. In fact one of the first applications of lasers was to the drilling of diamond dies for wire drawing, resulting in a reduction of the time involved in each operation from $12 \mathrm{~h}$ to $20 \mathrm{~min}$. With the greatly increased complexity of microcircuits requiring greater intricacy and closer tolerances, the speed, accuracy $(\sim 1 \mu \mathrm{m})$ and flexibility of laser machining is being more widely appreciated. Thus complex masks that take 4 days to make by conventional techniques can be cut in $10 \mathrm{~min}$. Computer control can easily be incorporated so that changes can be made rapidly and frequently.

\section{Optical-fibre Communications}

Perhaps, at long last, the greatest impact of optical electronics will be in the field of communications. From the very beginning in 1960 one of the most exciting possibilities was that of exploiting the potentially enormous bandwidth capability of lasers as carrier-frequency sources. The difficulties are well known. Propagation through the atmosphere is limited to distances of a few kilometres because of atmospheric turbulence, even in fine weather. Outer space is an ideal medium but the number of applications outside the Earth's atmosphere is always likely to be small. Techniques have been devised, with great ingenuity, for protecting laser beams from the vagaries of the atmosphere by enclosing them

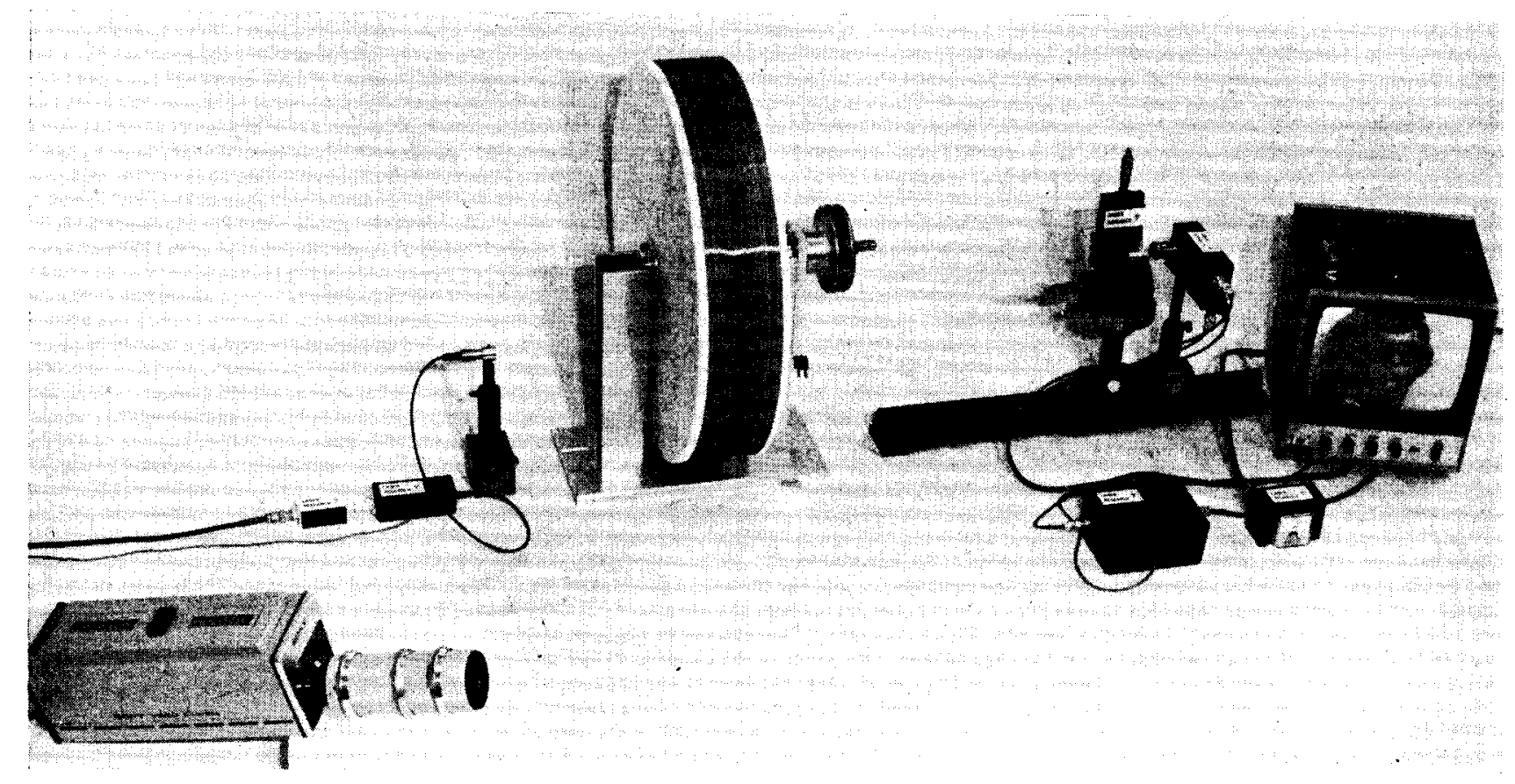

Fig. 3. Glass fibres will play an important part in long-distance communications systems of the future. The photograph shows the transmission of television pictures along a $1 \mathrm{~km}$ length of such fibre. Both the fibre and the link have been made in the Department of Electronics at the University of Southampton. This equipment was used for the first commercial application of fibres to television broadcasting when an entire colour television programme, from the Royal Institution, was passed through it en route to the transmitter. 

in pipes and correcting for the effect of spreading due to diffraction. ${ }^{10}$ Technically this can be done and transmission losses as low as $1 \mathrm{~dB} / \mathrm{km}$ have been achieved but the cost is considerable and quite uneconomic at the present time.

Another method of providing guidance, and of cunningly circumventing the problem of light travelling in straight lines, is to use a fibre consisting of a glass core having a high refractive index surrounded by a cladding of lower index. As early as August 1964, in an address to the British Association for the Advancement of Science, the author ${ }^{11}$ speculated on the use of light and glass fibres in the telephone network, instead of electric currents and wires, but developments did not start in earnest until publication of the classic article of Kao and Hockham $^{12}$ of STL in 1966 . At the time the problem seemed a formidable one; the attenuation of existing fibres was about $1000 \mathrm{~dB} / \mathrm{km}$, the bandwidth was expected to be low and fibre bundles were fragile. Since then enormous strides have been made resulting in fibre attenuations $^{13}$ of $2 \mathrm{~dB} / \mathrm{km}$ produced as a matter of routine, bandwidths of $1 \mathrm{GHz}$ in a $1 \mathrm{~km}$ length $^{14}$ of fibre having a diameter of $100 \mu \mathrm{m}$, and fibres coated with nylon which are too strong to be broken by hand. Such fibres are flexible and capable of being incorporated into simple but effective forms of cable. The bandwidth of a single fibre is much greater, and the attenuation lower, than existing copper coaxial cables and the diameter is considerably smaller. Thus the capacity of the present telephone network could be very greatly increased, with little additional installation expense, by the gradual introduction of optical fibre cables.

Trial installation cables of optical fibres have been laid in a number of countries and have given satisfactory and reliable service. Techniques for making joints and junctions are being rapidly developed while a fibre cable of, say, $1 \mathrm{~cm}$ diameter is more easily laid than a much

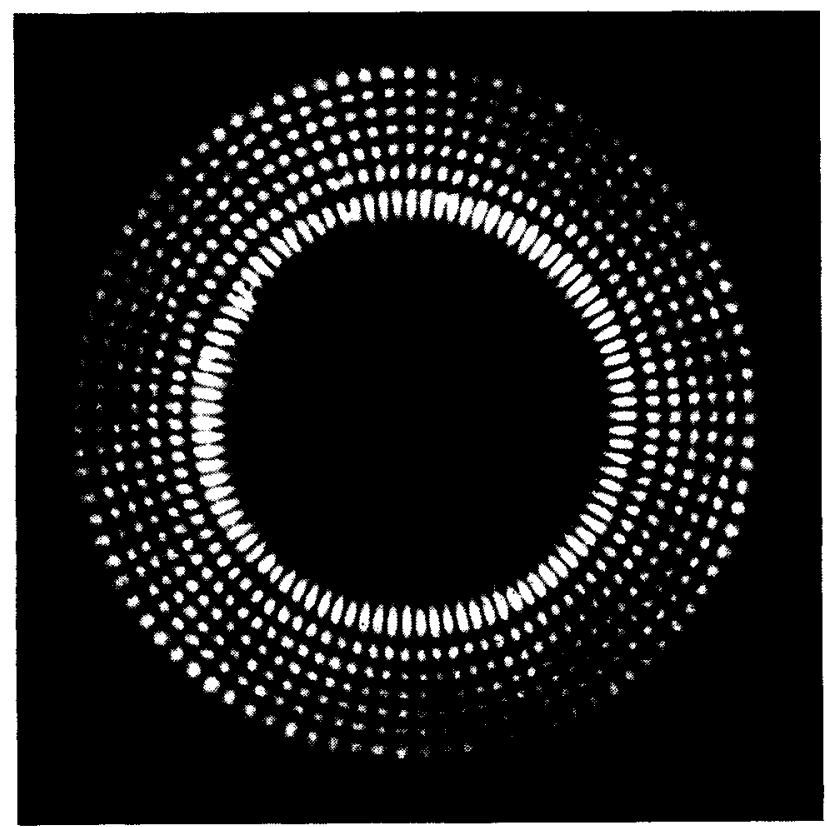

Fig. 4. High-order mode pattern of a multimode optical fibre. (By courtesy of Mr. W. J. Stewart, The Plessey Co. Ltd., Towcester.)

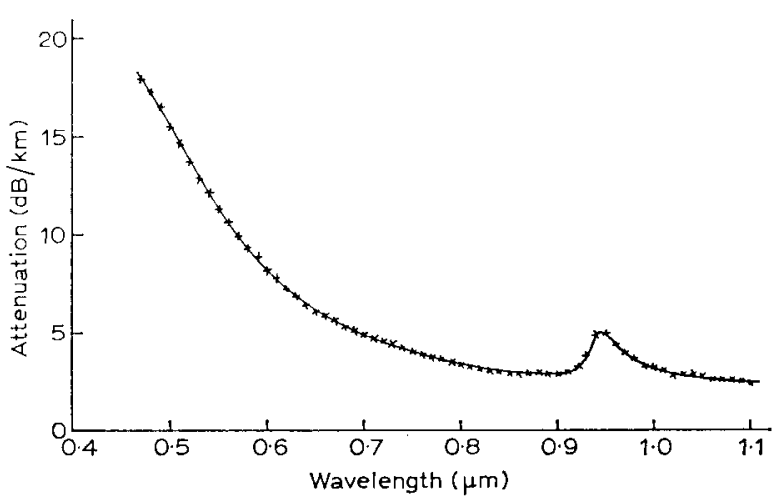

Fig. 5. Spectral attenuation curve of a $1 \cdot 2 \mathrm{~km}$ length of phosphosilicate optical fibre.

heavier $10 \mathrm{~cm}$ diameter copper coaxial cable. An optical fibre system will be simple in concept, with electrical signals turned into optical ones at the input and a reverse process at the output. Satisfactory detectors already exist but the lifetimes of present-day semiconductor lasers need a significant improvement. On the other hand, it has been shown ${ }^{15}$ that light-emitting diodes, which already exist with long lifetimes, could well be adapted to give the same bandwidth. Optical fibres will probably find their first application in special-purpose links but might be put into developmental trials for short links between local exchanges within five years.

\section{Conclusions}

The trend to higher frequencies received a tremendous impetus with the advent of the laser. However, technology can normally only cope with gradual improvements in techniques and cannot implement easily, or quickly, an advance in frequency capability of four or five orders of magnitude. Furthermore, the laser has to await the development of associated techniques before it can be properly used. For example, even if the cavity magnetron had been invented in 1938 radar as we know it would not have appeared overnight; it would still have been necessary to await the development of waveguide components, T-R cells, aerials, circulators, isolators and so on before the new device could be used properly in an effective system. In the same way, lasers cannot be fully exploited in isolation and are dependent on the creation of many other associated techniques and components. In fact it is necessary to think in terms of laser systems and the increasing number of applications in all fields is due, in part, to an appreciation of this factor.

The past fifteen years have shown tremendous promise and have provided exciting new experiments as well as some disappointments. Nevertheless, steady progress in optical techniques is being made and $\mathrm{I}$ am sure we shall look back on this period as the formative one for optical electronics.

\section{References}

1. Einstein, A., 'Zur Quantentheorie der Strahlung', Phys. Zeits., 18, pp. 121-8, 1917

2. Fabrikant, V. A., see B. A. Lengyel, 'Evolution of masers and lasers', Am. J. Phys., 34, pp. 903-13, 1966. 
3. Weber, J., 'Amplification of microwave radiation by substances not in thermal equilibrium', IRE Trans. on Electron Devices, No. PGED-3, pp. 1-4, June 1953.

4. Basov, N. G. and Prokhorov, A. M., 'Applications of molecular beams to radio spectroscopic studies of rotation spectra of molecules', J. Exp. Theor. Phys. (U.S.S.R.), 27, pp. 431-8, 1954.

5. Gordon, J. P., Zeiger, H. J. and Townes, C. H., 'Molecular microwave oscillator and new hyperfine structure in the microwave spectrum of $\mathrm{NH}_{3}{ }^{\prime}$, Phys. Rev., 95, pp. 282-4, 1st July 1954.

6. Bloembergen, N., 'Proposals for a new type solid-state maser', Phys. Rev., 104, pp. 324-7, 15th October, 1956.

7. Wilmshurst, T. H. and Gambling, W. A., 'An electron-spinresonance spectrometer using the ammonia maser as a preamplifier', Physics Letters, 5, p. 228, 1963.

8. Schawlow, A. L. and Townes, C. H., 'Infrared and optical masers', Phys. Rev., 112, pp. 1940-9, 15th December 1968.

9. Maiman, T. H., 'Stimulated optical radiation in ruby', Nature, 187, pp. 493-4, 6th August 1960.

'Optical maser action in ruby', Brit. Commun. Electronics, 7, pp. 674-5, September 1960.
10. Goubau, G. and Schwering, F., 'On the guided propagation of electromagnetic wave-beams', IRE Trans. on Antennas and Propagation, AP-9, pp. 248-56, 1961.

11. Gambling, W. A., 'Possibilities of optical communications', Engineering, 198, pp. 776-7, 18th December 1964.

12. Kao, K. C. and Hockham, G. A., 'Dielectric-fibre surface waveguides for optical frequencies', Proc. Instn Elect. Engrs, 113, pp. 1151-8, 1966.

13. Payne, D. N. and Gambling, W. A., 'Preparation of waterfree silica-based optical fibre', Electronics Letters, 10, pp. 289-90, 25th July 1974.

14. Payne, D. N. and Gambling, W. A., 'A borosilicate-cladded phosphosilicate-core optical fibre', Optics Communications, 13, pp. 422-5, April 1975.

15. Payne, D. N. and Gambling, W. A., 'Zero material dispersion in optical fibres', Electronics Letters, 11, pp. 176-8, 17th April 1975.

Manuscript received by the Institution on 7th July 1975. (Paper No 1673/Com. 117)

(C) The Institution of Electronic and Radio Engineers, 1975 